\title{
Temperature diagnostics with multichannel imaging telescopes
}

\author{
M.A. Weber, E.E. Deluca, L. Golub, and A.L. Sette \\ Harvard-Smithsonian Center for Astrophysics, 60 Garden St, Cambridge, MA 02138, USA \\ email: golub@head.cfa.harvard.edu
}

\begin{abstract}
.
Modern solar telescope design in the EUV to x-ray range is now capable of producing large images in multiple channels at rapid cadences, with high spatial and temperature resolution. We discuss reconstruction of differential emission measures for solar coronal plasma using two state-of-the-art instruments: the X-Ray Telescope on Solar-B, and the Atmospheric Imaging Assembly on the Solar Dynamics Observatory. We discuss the relative merits of iteration and direct inversion methods for determining $\operatorname{DEM}(T)$. We also consider strategies for automating and visualizing DEM maps, given the high data rates that these instruments will produce. We touch on the scientific potential of high-cadence, spatially resolved DEM data products.
\end{abstract}

\section{Introduction}

Solar telescope design in the EUV to x-ray range is now capable of producing large images $(4096 \times 4096$ pixels) in multiple channels (six or more) at rapid cadences $(\sim 10$ seconds), with high spatial (sub-arcsecond) and temperature (order of emission kernel widths) resolution. Such instruments permit more sophisticated temperature diagnostics than the filter-ratio method used with the previous generation of coronal imaging telescopes. In this paper, we discuss the issues, capabilities, and science for DEM reconstruction in the context of two state-of-the-art instruments: the X-Ray Telescope on the Solar-B spacecraft, and the Atmospheric Imaging Assembly on the Solar Dynamics Observatory. The X-Ray Telescope (XRT) is a grazing-incidence, broadband instrument with nine diagnostic filters spanning the range $6.1<\log T<7.5$. More information on the design of XRT for DEM reconstruction can be found in Golub et al. (2004).

The Atmospheric Imaging Assembly (AIA) is a normal-incidence, narrow-band instrument with four 20-cm, dual-channel telescopes operating simultaneously at a 10 second cadence (Fig. 1a). The mirrors in each telescope are subdivided into two areas (called "D"s) with different multilayer coatings, giving seven EUV channels, plus a channel used for UV and white-light observations (Fig. 1b). The AIA will be able to image coronal plasmas for $5.5<\log T<7.5$, and cooler regions of the solar atmosphere. The focal plane detector is a $4 \mathrm{k} \times 4 \mathrm{k}$ back-illuminated CCD with 0.6 arcsecond pixels and a 43 arcminute field-of-view which will be able to capture the entire solar disk - the AIA will combine the f.o.v. of SoHO/EIT with the resolution of TRACE.

\section{Two methods for DEM reconstruction}

The differential emission measure (DEM) is a basic observable of the coronal plasma in a region of interest $\mathrm{d} V$. The DEM is a measure of the amount of emitting plasma:

$$
\int n_{e}^{2} \mathrm{~d} V=\int \operatorname{DEM}(T) \mathrm{d} T
$$



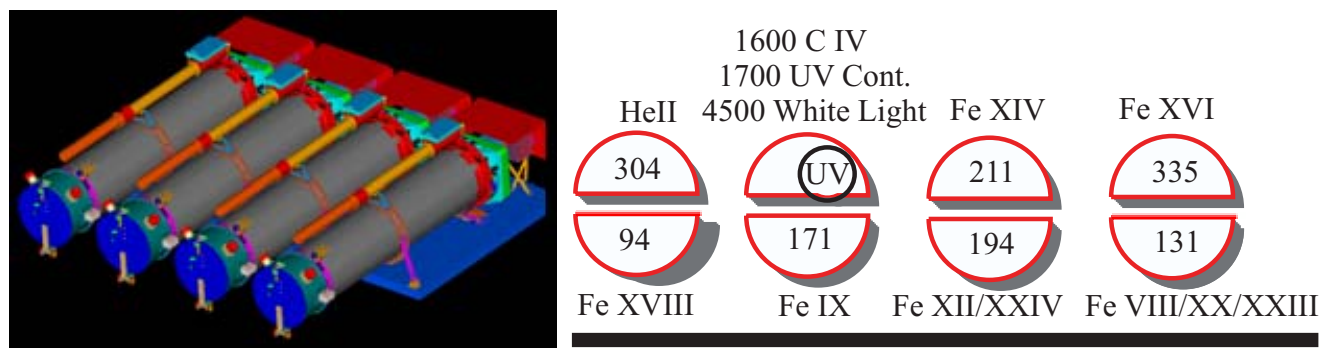

Figure 1. (a) Model of the AIA assembly. (b) Diagram showing the arrangement of the wavelength channels on the four telescopes. The UV passband has three separate channels available through the use of focal plane filters.

where $n_{e}$ is the electron number density. The intensity $d_{i}$ observed in channel $i$ is

$$
d_{i}=\int \operatorname{DEM}(T) r_{i}(T) \mathrm{d} T
$$

where $r_{i}(T)$ combines the known information, i.e., the temperature response function for channel $i$ with the plasma emissivity function. The DEM may be found, in principle, by inverting Eq. 2.2, but that is an ill-conditioned problem if the $r_{i}(T)$ overlap in temperature coverage (as they do for the XRT and AIA). We discuss two approaches to solving this inversion problem: an iterative search method, and direct inversion methods.

\subsection{Iterative methods}

We wish to find the "best" fitting DEM for a given set of observations in several spectral channels. Here we consider a set of images taken of an active region (AR). We assume that the structure of the AR loops is unchanged during the time it takes for the instrument to accumulate all of the images. Before processing, the set of images is co-aligned and reduced to physical units $(e . g ., \mathrm{DN} / \mathrm{s})$. In this section, we discuss how we estimate the DEM in a given pixel.

Our procedure produces an iterative least-squares fit to the observations using a DEM represented by a spline with evenly spaced knots in $\log T_{e}$ space. For the set of observations (all $i$ ) in pixel $p$, we wish to solve the simultaneous equations of Eq. 2.2. With the forward modeling approach, we assume a starting DEM, apply Eq. 2.2, and compare the predicted observations for each filter with the real observations. The optimal DEM spline is found using IDL mpfit routines from Craig B. Markwardt (http://cow. physics.wisc.edu/ ${ }^{c r a i g m / i d l / i d l . h t m l), ~ w h i c h ~ s e a r c h ~ t o w a r d s ~ a ~ m i n i m u m ~} \chi^{2}$ statistic by varying the spline knots. These routines use the non-linear least-squares method based on the MINPACK-1 routines (http://www.netlib.org/minpack/). This method, including uncertainty analysis, is described in more detail in Golub et al. (2004).

\subsection{Direct inversion methods}

Another class of solution methods is comprised of inversion algorithms. Eq. 2.2 can be written in matrix form as

$$
R_{i T} \operatorname{DEM}_{T}=d_{i},
$$

representing a simultaneous set of linear equations, where $\mathrm{DEM}_{T}$ is a vector of length $N_{T}$, and $d_{i}$ is a vector of length $N_{i}$. For $N_{T}=N_{i}$, the system is determined and there is a unique solution (assuming no degeneracies in the response functions). The inversion is easily solvable with standard linear algebra techniques (e.g., lower-upper decomposition). However, unless the selection of temperature bins (implicit in the response functions) 
happens to be closely consistent with the characteristics of the "true" DEM function, the solution DEM is often unphysical. For example, solution functions often show significant negative DEM values. Furthermore, the constraint $N_{T}=N_{i}$ may imply poor resolution in temperature.

For $N_{T}>N_{i}$, the system in Eq. 2.3 is underdetermined, and there is an infinite family of solutions. In other words, the a posteriori information (i.e., the data) is insufficient to constrain the model to a unique solution. In this case, there is freedom to constrain the solution with a priori information (i.e., physical constraints, such as assumptions about positivity or smoothness). Regularization methods (see Press et al., 1992) turn Eq. 2.3 into a well-posed minimization problem with the addition of such a constraint, by trading the quality of fit to the data against the quality of fit to the assumed constraint. (The amount of trade-off is typically adjusted such that the $\chi^{2}$ fit to the data is $\sim N_{i}$.) Note that this approach requires an explicit choice of a priori assumptions.

Without making any a priori assumptions, the method of singular value decomposition (SVD) can be used to describe the family of solutions to Eq. 2.3. If $R_{i T}$ is considered as a linear map from the DEM space of $N_{T}$ dimensions to the data space of $N_{i}$ dimensions, then the SVD algorithm provides the $N_{i}$ DEM orthonormal basis functions which solve Eq. 2.3. This reduces the inverse problem to a square system of equations with a determinable principal solution. SVD also provides $\left(N_{T}-N_{i}\right)$ DEM orthonormal null-space functions, which add to the principal solution to generate the infinite family of solutions to Eq. 2.3. (Note that there is no physical significance inherent to the principal SVD solution, although it happens to be the solution vector of shortest norm.)

\section{Comparing DEM reconstruction methods}

We compared the iterative and SVD (inversion) methods to evaluate their pros and cons for implementation of an automated algorithm. A variety of nine input DEMs were designed and folded through the AIA response functions to produce simulated observations. Then each set of observations was solved for a reconstructed DEM using three methods: (a) "Simple" inversion (where $N_{T}=N_{i}$ ); (b) SVD principal inversion; and (c) the iterative approach. The "Simple" inversion method was included as a baseline for comparisons, even though it was expected to produce relatively poor reconstructions. We used six of the AIA's EUV channels $\left(N_{i}=6\right)$, and solved for DEMs with $N_{T}=19$ temperature bins spanning the range $\log T=5.6-7.4$.

Fig. 2 shows four of our model DEMs and the respective solutions for the SVD and iterative methods. (Note that model " $\mathrm{h}$ " is an empirical active region DEM, derived from observations by Dupree et al., 1973). These plots illustrate typical aspects of the solutions. The SVD and iterative methods do about equally well at reconstructing the DEMs (this is quantified below). For input models which have a constrained temperature range, the solutions accurately recover the central temperature. There is a lower limit on the width (resolution) of the fits due to the width of the temperature response functions. The iterative method always gives positive solutions, while the SVD method often produces solutions with small negative components.

We have quantified three measures of the relative value of the three solution methods considered. These are recorded in Table 1. The first quantity is runtime. For each set of observations, we calculated the average runtime of the method for 100 runs on a Sun Ultra 10 workstation, with a $440 \mathrm{MHz}$ processor and $1.0 \mathrm{~GB}$ of RAM. As expected, the inversion methods are substantially faster than the iterative approach: $\tau$ (iterative) $\sim 400 \times \tau(\mathrm{SVD}) \sim 10^{4} \times \tau($ "Simple" inversion $)$. 

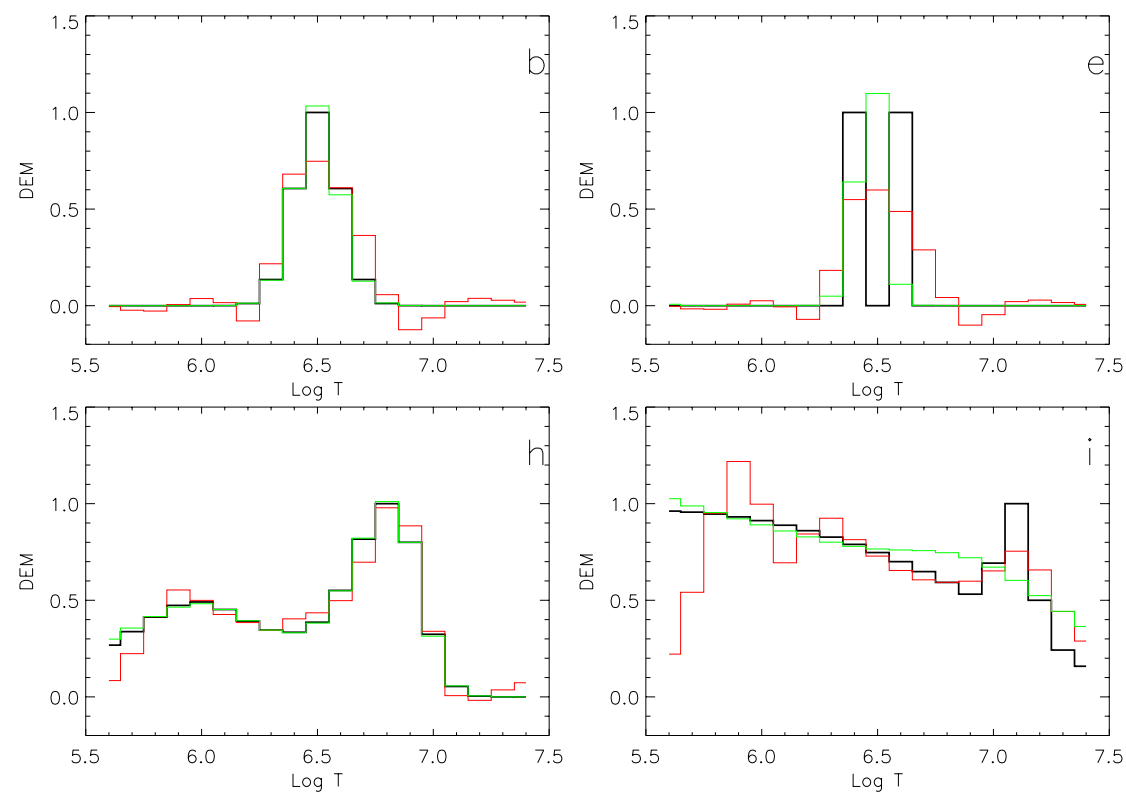

Figure 2. Four selected DEM models from a set of nine. (The letters correspond to entries in Table 1.) The thick black line is the model DEM; the dotted line is the estimate by the iterative method; and the dashed line is the SVD principal solution (direct inversion).

\begin{tabular}{c|cccccccccc|c}
\hline \hline DEM model $\Rightarrow$ & $\mathrm{a}$ & $\mathrm{b}$ & $\mathrm{c}$ & $\mathrm{d}$ & $\mathrm{e}$ & $\mathrm{f}$ & $\mathrm{g}$ & $\mathrm{h}$ & $\mathrm{i}$ & Median \\
\hline \hline Table 1.1: Runtimes (milliseconds) & & & & & & & & & \\
\hline "Simple" inversion & 0.055 & 0.054 & 0.053 & 0.053 & 0.055 & 0.056 & 0.055 & 0.054 & 0.054 & 0.054 \\
SVD & 1.3 & 1.4 & 1.4 & 1.4 & 1.4 & 1.4 & 1.3 & 1.3 & 1.3 & 1.4 \\
Iteration & 1800 & 590 & 750 & 750 & 570 & 510 & 1100 & 520 & 440 & 590 \\
\hline \hline
\end{tabular}

Table 1.2: $\chi^{2}$ between observations from model and from estimated DEM

\begin{tabular}{c|ccccccccc|c}
\hline "Simple" inversion & 0 & 0 & 0 & 0 & 0 & 0 & 0 & 0 & 0 & 0 \\
SVD & 0 & 0 & 0 & 0 & 0 & 0 & 0 & 0 & 0 & 0 \\
Iteration & 0.10 & 0.11 & 0.003 & 4.3 & 19.6 & 0.038 & 3.2 & 0.009 & 67.4 & 0.11 \\
\hline
\end{tabular}

Table 1.3: Cross-correlation coeff. between model DEM and estimated DEM

\begin{tabular}{c|ccccccccc|c}
\hline "Simple" inversion & 0.75 & 0.063 & 0.098 & 0.54 & 0.048 & 0.069 & 0.22 & -0.096 & 0.053 & 0.069 \\
SVD & 0.89 & 0.95 & 0.92 & 0.67 & 0.72 & 0.69 & 0.54 & 0.99 & 0.95 & 0.89 \\
Iteration & 1.00 & 1.00 & 1.00 & 0.56 & 0.42 & 0.64 & 0.48 & 1.00 & 0.99 & 0.99 \\
\hline \hline
\end{tabular}

Table 1. Quantitatively comparing DEM reconstruction methods for different DEM models. Models $\{\mathrm{a}, \mathrm{b}, \mathrm{c}\}$ are Gaussian distributions for cool, warm, and hot plasma. Models $\{\mathrm{d}, \mathrm{e}, \mathrm{f}\}$ are "double isothermal" peaks for cool, warm, and hot plasma. Model g is a cool isothermal peak plus a hot isothermal peak. Models $\{\mathrm{h}, \mathrm{i}\}$ are multithermal and shown in Fig. 2. The median values are simply the medians for this arbitrary set of nine models. 
The second tabulated quantity is the $\chi^{2}$ fit to the observations. Note that inversion methods reproduce the observations perfectly. This is a mathematical consequence when solving a well-determined (or underdetermined) system of equations. Iteration converges to the first minimum it reaches from the starting guess, which may only be a (nonzero) local minimum in $\chi^{2}$.

The third tabulated quantity is the cross-correlation coefficient between the input and solution DEMs. The iterative method is shown to most accurately reconstruct the DEMs, typically. "Simple" inversion does not have the temperature resolution to accurately reproduce the input DEMs. The SVD method does a fair job, considering that the principal solution is only one of a family of solutions, some of which might be much better. (In fact, since we are solving over the same temperature bins for which the input DEMs are defined, the input DEM is definitely a member of the SVD family of solutions. Of course, this would not be expected when analyzing observations of coronal plasma.)

From these results, it is apparent that the iterative approach does well at reproducing our sample of model DEMs, giving positive solutions. Its ability to reproduce the observations (in terms of the $\chi^{2}$ statistic) varies greatly. The inversion methods are much faster, which is a prominent concern when designing a DEM data product for, e.g., the AIA, which produces images with $16+$ Mpix at a cadence of 10 seconds. The SVD principal solution reproduces the observations perfectly (before error analysis), and does almost as good a job at reconstructing the DEM models as the iterative method does, although it can return negative components. Note that there is freedom to impose some physical constraints on inversion methods (such as positivity) while retaining computation speeds, by allowing the $\chi^{2}$ statistic to go to $N_{i}$.

\section{Sixteen Million DEMs Every Ten Seconds}

Calculating DEMs at several points in an image is straightforward for nearly any method, however the true power of AIA and XRT DEM observations comes when the DEMs are available for every image set. Say, for example, there is a coronal loop visible in a few adjacent channels, and we wish to test the hypothesis that the loop is isothermal. With AIA standard cadence we can follow the evolution of this loop from appearance to disappearance with a ten second cadence. If the loop lasts for 100 minutes we will have an observational dataset that consists of 600 sets of 6 EUV images to form DEMs from. For this project we would limit our analysis to the few hundred pixels surrounding the loop, say $1 / 4$ million pixels/frame total. It is apparent that if the visible loop is in fact a physically distinct structure in the corona, its evolution will be different from the surrounding plasma. There will be "features" within the loop's DEM that will evolve together; the rest of the DEM in the loop can be attributed to changes along the line of sight, not associated with the loop in question. An isothermal loop will have a DEM feature that is within the temperature resolution of the telescopes used $\left(\sim 0.2 \log T_{e}\right)$. The ability to calculate DEMs at every point along a loop (and all adjacent points, too) will provide an unprecedented set of observational constraints on coronal loop models and corona heating theories.

In this section we discuss our investigations of pre-conditioning methods, which optimize DEM reconstruction for the iterative approach by making an informed initial DEM guess that takes advantage of the sharing of DEM information among the pixels in an image. In addition, we evaluate the effectiveness of different DEM display options, including single temperature emission maps and time-progression DEM movies. We aim to construct DEMs efficiently and display complex information in a concise, useful manner.

We use a simulated cube of coronal plasma, with dimensions $79 \times 137 \times 137$ (z,x,y), to model DEM reconstruction from AIA observations. (The model is provided by Gudiksen 


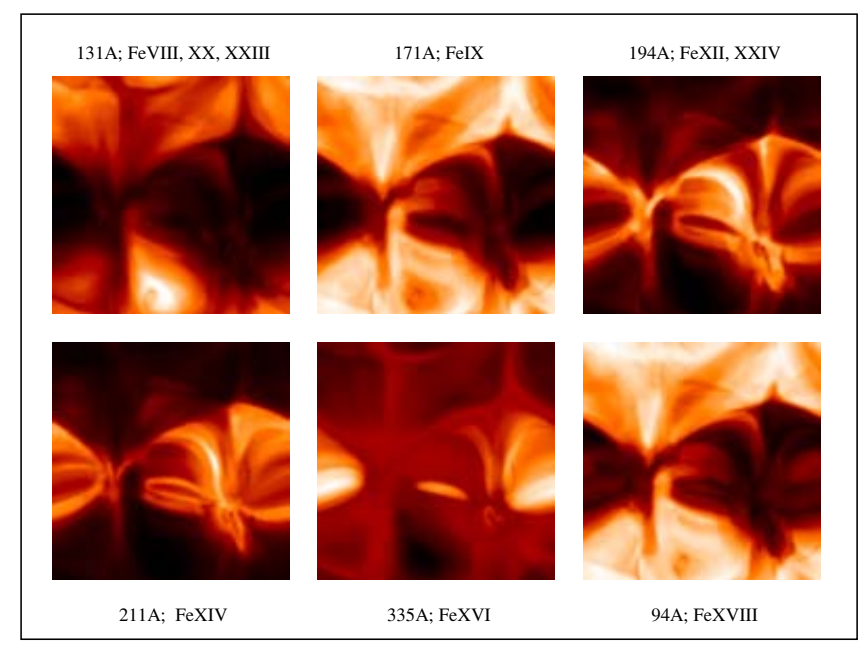

Figure 3. AIA simulated observations of the model, top view.

et al., 2001, 2004.) The original simulation had a $150 \times 150 \times 150$ cube $\times 45$ time frames, with $n_{e}=10^{8}-10^{17} \mathrm{~cm}^{-3}$ and $\log T=3.6-6.6 \mathrm{~K}$. From the cube we constructed a DEM, at each pixel, along a vertical line-of-sight. We binned the data in temperature (bins of $\log T=0.1)$, and interpolated to match AIA pixel scale $(440 \mathrm{~km})$. Observations were generated by convolving the DEMs with a spectral model and the AIA response function for each channel (Fig. 3).

\subsection{Optimization methods}

As shown in Table 1.1, the run times for SVD are $\sim 400$ times faster than the iterative method. However, the iterative method provides a better fit to the DEMs. The iterative model depends on an initial guess of the DEM; currently we are using a flat (i.e., an uninformed) guess. We can improve the runtime for the iterative method by using an informed guess. Four methods are compared: Brute Force - our standard method; for each image, solve for the DEM of each pixel independently using a flat first guess. Single Average - for each image, average all pixels and calculate a "global" DEM; use this as a first guess for the DEM reconstruction of all pixels. Time Dependent - for the first image, solve for the DEM of each pixel independently using a flat first guess; use the resulting DEM image as a first guess for calculations in second image; iterate to last image. Time Averaging - for first image, use a global DEM as a first guess for DEM reconstruction for each pixel; use the resulting DEMs as a first guess for calculations in second image; iterate to last image. To compare computation times from method to method, we performed a series of reconstructions for 10 time frames of simulated data with eight different square array sizes using each of the above methods.

\subsection{Optimization results}

The results are shown in Fig. 4: a log-log plot of total number of pixels vs the computation time. For the Brute Force method, computation time goes roughly as $N^{2}$, where $N$ is the square array length. For the Single Average method, there are improvements for small array sizes, but when the array grows too large, the global guess is no longer a good approximation for most pixels. The Time Averaging and Time Dependent methods improve the convergence time by nearly a factor of three. Note that pre-conditioning 


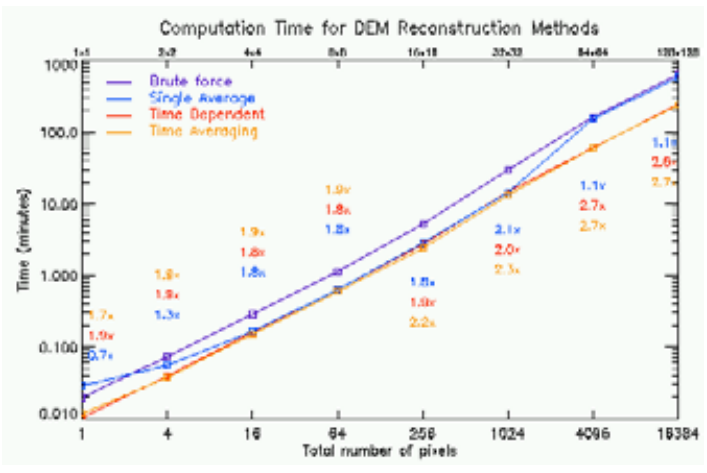

Figure 4. A log-log plot showing the computation time as a function of the total number of pixels in the image.

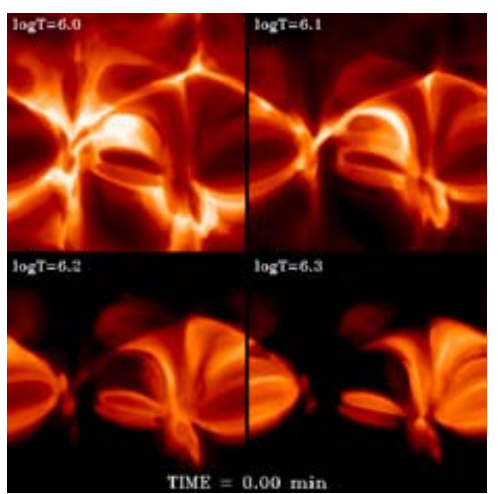

Figure 5. Single temperature images constructed from DEM solutions.

does not sacrifice goodness of fit for speed (iterative DEM solutions are surprisingly independent of initial guess).

While we expect to use pre-conditioning to calculate DEMs for large data sets, a factor of three improvement is not sufficient. The SVD fits described above give improvements of a factor of four hundred. We will look into improving the accuracy of SVD type methods without sacrificing speed.

\section{How do we view the data?}

It is unlikely that the AIA and XRT missions will be able to provide DEMs at every pixel for every set of observations. A standard data product might be isothermal images of the corona constructed every hour. Fig. 5 shows examples of this data product for four temperatures, $\log T=6.0-6.3$. The tools to construct DEMs from time dependent data sets are essential. We have explored different ways of presenting the results of that analysis: movies of single temperature images (Fig. 5), movies of observations with DEMs from selected points displayed (Fig. 6, top), and movies of DEM differences (DEM $\left.\right|_{t_{n}}$ - DEM $\left.\right|_{t_{n-1}}$ ) (Fig. 6, bottom). Each of these have advantages and limitations - an isothermal loop should be easily seen in the single- $T$ movies, but if you want to understand the process of chromospheric evaporation, DEM differences may be the best approach.

\section{Conclusion}

The XRT and AIA instruments will provide solar scientists with an unprecedented set of observations. The volume of data and the complexity of DEM analysis place strong requirements on the data analysis system. Tools must be provided for: quick-look single temperature maps (based on DEM analysis); detailed DEM analysis of events by scientists, with error estimates; and plasma emission codes with variable parameters and keywords that track the assumptions made in the DEM processing. These tools will need to be evaluated on various criteria (e.g., speed, confidence intervals, fit to observations), depending on the particular questions being put to the data. The advances currently happening in emission line codes, imaging solar telescope design, and DEM reconstruction techniques shall address these issues.

\section{Acknowledgements}

The authors would like to thank Boris V. Gudiksen and Åke Nordlund for providing the simulated coronal data analyzed here. This work is supported under AIA contract SP02D4301R to Lockheed Martin Corp., and under XRT contract NAS8-01003 to NASA. 

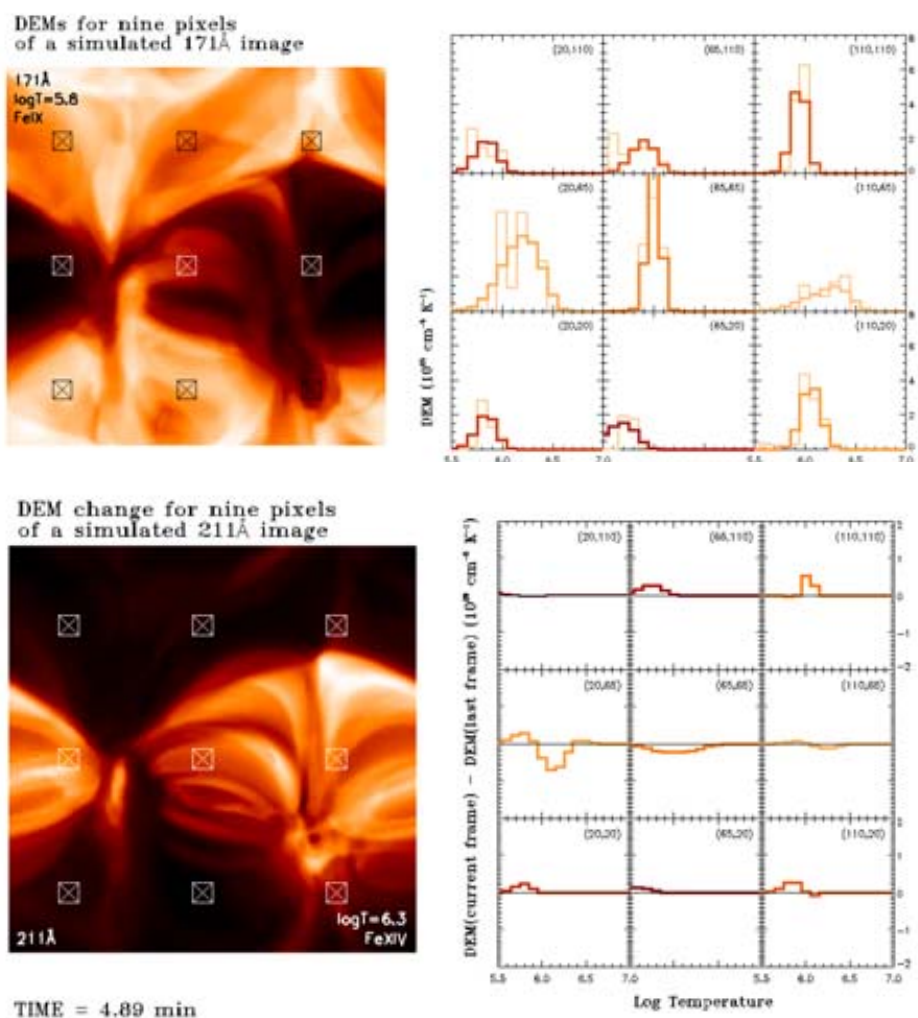

Figure 6. (Top) An Fe IX observation with DEM plots at 9 selected points in a simulated $171 \AA$ image. The model DEMs (lighter line) and estimated DEMs (darker line) are both plotted. (Bottom) An Fe XIV observation with DEM differences at 9 selected points in a simulated $211 \AA$ image. The curves show at which temperatures the density is increasing (positive) and decreasing (negative).

\section{References}

Dupree, A.K., et al. 1973 ApJ 182, 321.

Golub, L., Deluca, E.E., Sette, A., \& Weber, M. 2004 In Proceedings of the Fifth Solar-B Science Meeting (ed. T. Sakurai \& T. Sekii, ASP Conf. Ser.), in press.

Gudiksen, B.V. 2004 The Coronal Heating Problem Ph.D. thesis, Dept. of Astron., Stockholm University.

Gudiksen, B.V., \& Nordlund, Å. 2001 ApJ 572, L116.

Press, W.H., et al. 1992 Numerical Recipes (Cambridge: Cambridge Univ. Press). 